\title{
Influenza and norovirus outbreaks in an inpatient mental health setting: Analysis and strategies for successful containment
}

\author{
Amber L. Linkenheld-Struk, BA, MLT, CIC; Natasha Salt, BSc, BASc, CIC; Jackie Griffin-White, BSc, RN, MSN \\ Sunnybrook Health Sciences Centre, Toronto, ON, Canada
}

Corresponding author:

Amber Linkenheld-Struk, BA, MLT, CIC, Infection Prevention and Control, Sunnybrook Health Sciences Centre, Toronto, ON, Canada, M4N 3M5 Tel: 416-480-6100 x7524 amber.linkenheld-struk@sunnybrook.ca

\begin{abstract}
During the spring of 2019, an inpatient psychiatry unit at a large tertiary care hospital experienced two outbreaks between April 6 and May 7, first influenza A, with seven identified cases, followed by norovirus with three identified cases. This outbreak investigation examines the management of both outbreaks and highlights unique

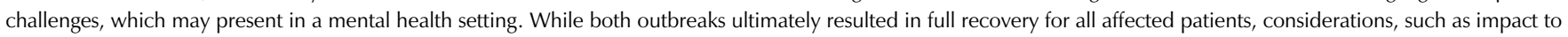
patient therapies, fomite transmission, shared spaces, access to psychiatric services and impact to mental health require innovative thinking. Unique outbreak management considerations, and strategies are examined.
\end{abstract}

\section{INTRODUCTION}

Despite the distinctive challenges posed to Infection Prevention and Control (IPAC) in a mental health setting, there is a dearth of research dedicated specifically to infection mitigation strategies in these areas. In light of this, challenges may arise in applying accepted standards for outbreak control when the situation presents itself, without clear guidance on alternative avenues for effective control.

During the spring of 2019, two discrete outbreaks occurred within the same inpatient psychiatric unit in a large Toronto tertiary care facility. This outbreak report will cover both an influenza A outbreak that was declared on April 6 and ended on April 17, 2019, and a norovirus outbreak that was declared on May 1 and ended May 7, 2019. This outbreak report will outline the course of these outbreaks, demonstrate that the inpatient psychiatric setting must be considered as a unique environment for outbreak management, requiring flexible mitigation strategies to support standard outbreak protocols, and attempt to demonstrate some broadly applicable strategies for all inpatient mental health settings.

\section{Case definition/identifications}

In both instances, cases were reviewed by IPAC professionals and outbreaks were declared compliant with Ontario Ministry of Health and Long-Term Care (MOHLTC) guidelines [1,2].

Nurses on the unit performed daily syndromic surveillance and contacted IPAC directly via phone or page if there were cases of concern and chart review and nursing interviews were conducted by IPAC to better typify the symptoms. For the influenza outbreak, cases were identified by both symptom presentation and laboratory confirmation through positive midturbinate (MT) swabs, which were tested by multiplex polymerase chain reaction (PCR) against a respiratory virus panel at an in-house laboratory. Norovirus samples were tested by the Public Health Ontario Laboratory (PHOL) via viral culture, but as results were returned after the outbreak was declared over, cases were line listed exclusively based on symptomatic presentation and the outbreak was managed as norovirus-like illness. As of November 2019, the PHOL changed to molecular testing for enteric viruses, but at the time of this outbreak, viral culture was in use [3].

\section{OUTBREAK DESCRIPTION \\ Setting}

The inpatient psychiatry unit consists of 35 inpatient beds broken down into 22 adult beds, five psychiatric intensive care beds (PICU) and eight adolescent beds. Within the adult unit, there is only one room designed for single occupancy. These areas are geographically linked, without corridors dividing them, but split by semi-restricted doors, which limit, but do not eliminate patient movement between rooms. Washrooms are divided by gender, and are shared 


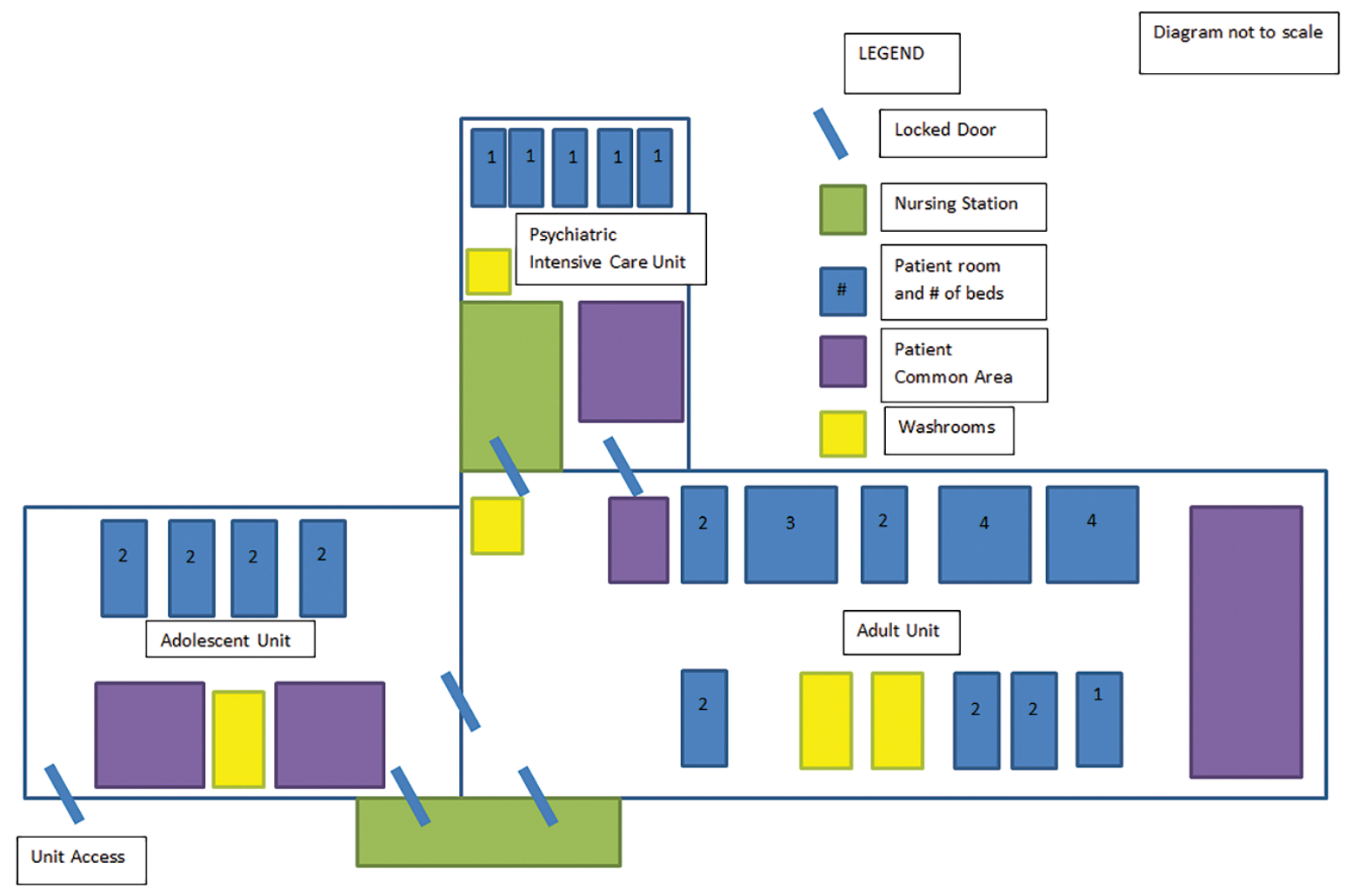

FIGURE 1: Physical layout of the inpatient psychiatry unit

stall-style spaces within the adult and adolescent areas with a single washroom being available on the PICU. All areas have shared spaces for patients, and PICU patients may typically access shared spaces in the adult area with a "pass", acuity dependent. The nursing station is linked between the adult and adolescent sides, and there is a single patient access to the entire unit via the adolescent area (Figure 1). Both outbreaks originated in the adult area, and the norovirus outbreak remained contained there.

The common areas for patient gathering and interaction as well as shared washrooms and dearth of single-patient rooms present as additional challenges in managing outbreaks as these features are not often present in standard acute care inpatient settings. Outside of mental health, there are generally no areas of patients to gather and socialize within the unit, and rooms have built-in toileting facilities and a larger number of private spaces are available. While this design can be acceptable due to increased patient mobility, and even necessary given the nature of treatments being received, it becomes extremely problematic when trying to contain a transmissible pathogen.

Like other acute care units, the inpatient mental health unit exists as part of a portfolio covered by an onsite IPAC professional, and is reviewed daily on weekdays for new cases of concern, and has 24 hours a day/7 days a week access to IPAC on call during off hours. This facilitates direct reporting occurring in a timely manner.

\section{Influenza A}

For the purposes of this outbreak, based on patient presentation, the case definition for the outbreak was established to be "A patient/resident or staff member with new onset of one or more of the following symptoms: fever, cough, runny nose, sore throat, hoarseness, congestion, shortness of breath (SOB), myalgia, or with confirmed laboratory results."

On March 30, a patient was admitted to the adult unit and within
72 hours had developed influenza-like symptoms. The patient was placed on droplet and contact precautions on April 2, and was found to have influenza A (H3N2). The morning of April 6, four additional nosocomial cases were identified with symptom onset greater than 72 hours after admission, presumably due to exposure to the community case (Figure 2).

All symptomatic patients were placed on droplet and contact precautions. Every effort was made to cohort symptomatic patients, and due to limitations in unit design, patients who could not be cohorted were placed on bed space precautions.

MT swabs were collected on all presenting patients, and testing was performed. Three of the four cases returned positive on April 6 for influenza A ( $\mathrm{H} 3 \mathrm{~N} 2)$, with no co-infecting viruses identified, and the fourth patient was negative for all respiratory viruses, however, they remained line listed due to casecompatible symptom presentation. An outbreak was declared on April 6, 2019. 


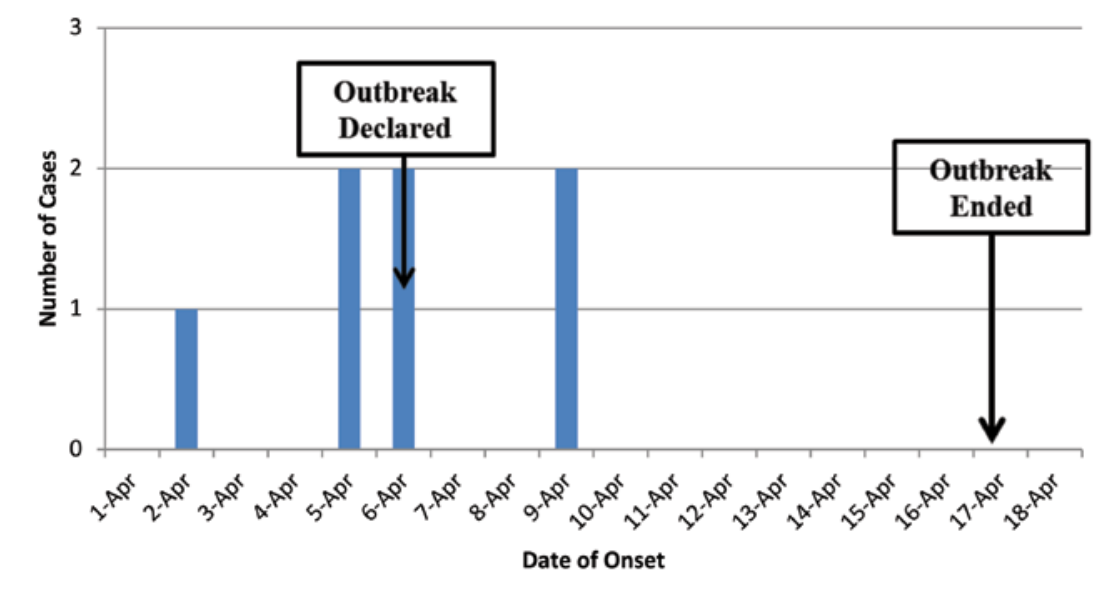

FIGURE 2: Influenza outbreak epidemic curve

As all cases had presented within the adult population, the decision was made to leave the adolescent area and PICU open to admissions, however, eliminate passes from PICU into the adult area. The adult unit was closed to admissions completely.

Oseltamivir treatment was offered to all cases and prophylaxis was offered to all exposed patients on the adult unit, as well as in the PICU, but not on the adolescent unit, as the risk was deemed low. One patient who was influenza positive declined to take treatment with oseltamivir, and one exposed patient declined prophylaxis.

On April 9, 72 hours after the outbreak was declared, two further symptomatic cases were identified. One was in the adult side, the patient who had declined to take prophylaxis, and one in the adolescent side. Both tested positive for influenza A (H3N2) by PCR. There were no sick visitors or staff identified on the adolescent side, thus the outbreak was geographically extended to include all areas in the psychiatry unit given this evidence of transmission.

No further transmission was noted after this point, and the outbreak ended eight days later, consistent with public health guidelines [1]. The attack rate among patients was $20 \%$ (7/35), and no staff or visitors reported symptoms during the period of the outbreak.

\section{Norovirus}

On May 1, 2019, IPAC was called with notification that two patients had experienced acute onset of copious vomiting and diarrhea (Figure 3). No other patients or staff reported illness, and the patients had not shared any common foods different than those served to the rest of the unit from hospital food services. Neither patient had an alternate explanation for the symptoms (i.e. withdrawal, medication change). As there was only one private room on the unit, the two affected patients were cohorted and placed on contact precautions.

Based on the presentation, an outbreak was called of norovirus-like gastrointestinal illness on the same day and the adult unit and PICU were closed to admissions. Environmental services staff were engaged to clean all bathrooms on the unit, and then a single washroom adjacent to the room of the affected patients was dedicated to symptomatic individuals. One patient who was discharged home on the date of the outbreak declaration called to inform the unit that he developed symptoms the day after his discharge on May 2. No further patients or staff on the unit developed symptoms and the outbreak was declared over on May 7. The attack rate was $14 \%(3 / 22)$ of admitted patients.

Lab results for viral culture returned from the PHOL after the outbreak had been declared over, and confirmed both patients, who were symptomatic on the unit, were positive for norovirus.

\section{DISCUSSION}

During the course of both outbreaks, unique considerations arose that were anticipated and unanticipated, some systemic issues, and some due to the population.

\section{Infrastructure, supplies and environmental cleaning}

At the outset of the influenza outbreak, it was found that the disinfectant wipes accessible to staff on the unit for equipment cleaning were still quaternary ammonia-based cleaners, as opposed

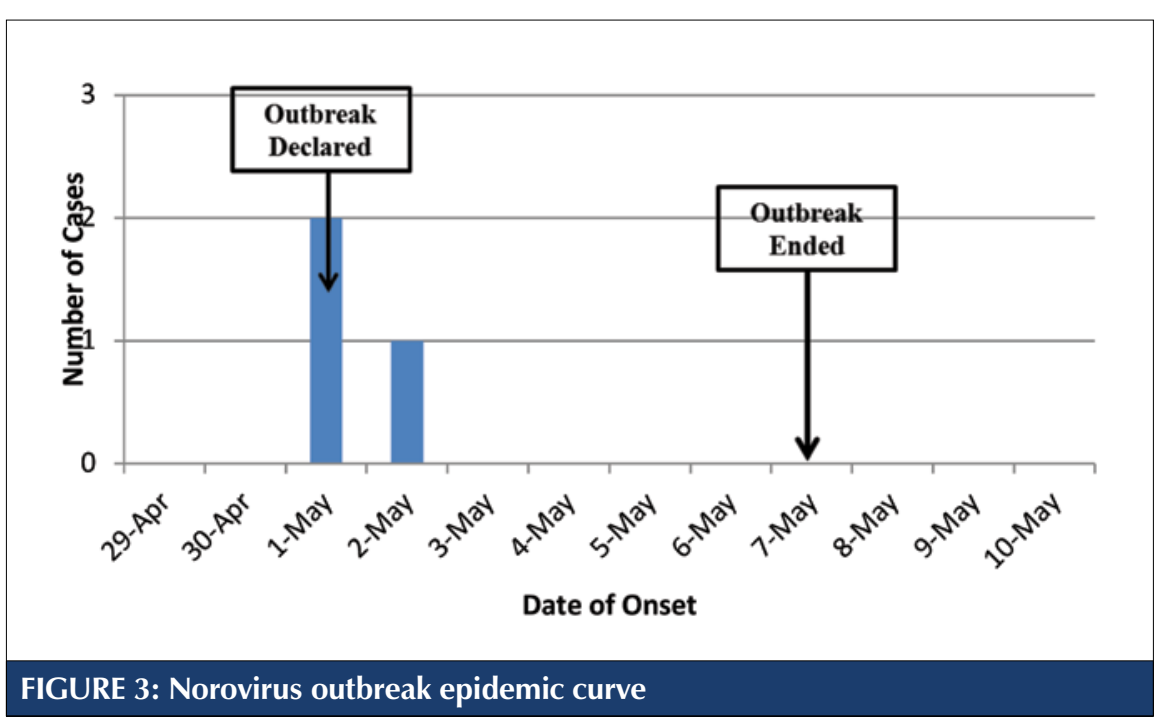


to hydrogen-peroxide-based cleaners available throughout the rest of the acute care areas of the facility. The transition to the hydrogen-peroxidebased cleaner was made immediately, and additional environmental services staffing was provided to focus on hightouch surfaces and shared spaces until the outbreak was declared over as required by MOHLTC guidelines.

The additional cleaning was particularly pertinent during the norovirus outbreak, where the ambulatory patient population and shared washroom spaces made transmission especially high risk. Games, books and any other non-wipeable objects were removed temporarily from patient common areas, to try and reduce the risk of fomite transmission.

At the outset of each outbreak, stored personal protective equipment (PPE) was in minimal supply, as procedures and interactions that would require PPE (line insertions, peri-care, wound dressings, etc.) are not generally needed or performed in the psychiatric setting at our facility.

Fortunately, amid the population admitted at the time, there was no concern for any patients consuming alcohol-based hand rub, thus supplies for hand sanitizer were available throughout the unit.

\section{Staff}

Collaborating with staff and senior leaders was important to successfully manage these outbreaks. Routine huddles and meetings supported active discussions about patient management, system challenges, staffing and environmental cleaning. They provided an opportunity to identify risks early and likely contributed to the low attack rates in both outbreaks.

When the influenza outbreak was declared, unit staff working on the weekend were unfamiliar with where to order or obtain additional PPE, which required hands on facilitation immediately after the outbreak meeting.

Similarly to how concerns arose with PPE, staff was not familiar with sample collection and test ordering protocols for norovirus testing and MT swabs, which again required direct guidance from IPAC to ensure samples were ordered appropriately.
Interestingly, these concerns around PPE and staff educational needs are an echo of the Gilbride et al 2009 paper, which examined a norovirus outbreak in an inpatient psychiatric unit, where they also described a lack of available PPE and staff knowledge as a barrier to effective outbreak control implementation [4]. The recurrence of this need across facilities seem to identify a gap in staff training that could potentially risk further outbreak propagation, or simply belay a lack of familiarity with the best way to manage patients on additional precautions for infection.

Specifically of concern for influenza, at the outset of the outbreak, vaccination rates among nursing staff on the unit were at $44 \%$, well below the target of $80 \%$ set by the Government of Canada designed to ensure patient safety [5], and below the institutional average of $69 \%$ achieved during the 2018/2019 influenza season. Occupational Health and Safety attended to administer influenza vaccination and dispense oseltamivir prophylaxis to staff who had not yet been vaccinated for the 2018/2019 season. Any staff who declined vaccination were restricted from working on the unit for the duration of the outbreak, but the preventative benefit of high vaccination rates among staff had already been lost.

\section{Patients}

The patient population in a psychiatric inpatient setting faces unique challenges in outbreak management. As with an outbreak in any setting, a patient's admitting diagnosis can put individuals at greater risk, but behaviours within the population can magnify both the risk of adverse outcome, the behaviours that lead to acquisition, and increase difficultly of true case identification. Outbreaks and the associated restrictions present psychological stressors for any admitted patient, and within the course of our outbreaks, there was concern for patients reporting symptoms they did not objectively have (never observed by nursing staff), patients actively trying to infect themselves, patients who had adverse psychological reactions to the closure of the unit areas resulting in harm (i.e. refusing to take medications, physically attacking the environment resulting in harm and damages), and patients with magnified symptoms of paranoia resulting from back-to-back outbreaks. In these circumstances, outbreak propagation can occur as a direct result of patient behaviours [6].

In-house activities for asymptomatic patients were not suspended, neither were most common areas closed during either outbreak, though patients were no longer allowed to access shared food storage areas and had to request personal food be accessed by staff. Some services facilitated by volunteers or therapists who attended multiple sites, such as art therapy, were suspended for the duration of the norovirus outbreak to avoid spread between facilities. Vaccinated staff and volunteers were permitted to remain during the influenza outbreak.

Patients who were ill could not attend group therapy sessions as required for treatment, thus treatment interventions were limited in a way they typically are not in other settings.

IPAC conducted a town-hall-style meeting with all patients at the outset of the norovirus outbreak to explain the situation, answer questions, and attempt to allay concerns about the outbreak and educate patients on the best ways to remain protected. This approach was deemed a highly effective method of communication as patients were engaged from the outset of the outbreak to ensure consistent messaging and inclusion of patients in decisions that affect them.

\section{Area mental health network}

Beyond unit level concerns, the outbreaks in this setting also had major system level impacts for the institution, and indeed the mental healthcare network in the Greater Toronto Area. Because psychiatric patients cannot be bed spaced to other available beds within the hospital, and the unit was closed, ambulances with psychiatric patients had to be redirected and 
when this was not possible, resulted in patients waiting extended periods in the emergency department until space could be found for them. Daily meetings with the network reviewed outbreak status and bed availability. Attempts were made to safely reopen a segregated area of the unit during the influenza outbreak after there had been 72 hours with no new cases, however, while this did not result in transmission, there was escalated behaviour in some at-risk patients due to new restrictions to movement around the unit.

\section{CONCLUSION}

Implementation of standard outbreak management protocols in our mental health setting presented some challenges. In particular, the set-up of the unit presented significant barriers to properly placing patients on additional precautions with use of a dedicated bathroom.

Key strategies that were effective in managing outbreaks in this setting included the town hall meeting with patients, increased presence of IPAC staff on the unit to guide staff in refreshers on personal protective equipment, sample collection and case finding, as well as removal of shared objects such as books and games which could easily serve as fomites. Enhanced cleaning also required more active following of ill patients who had to ambulate to shared bathrooms, rather than following a regular cleaning schedule. Furthermore, examination of behaviours and risk factors of the entire patient population were essential in order to allow for safe closure of a unit, a factor not typically considered in standard acute care settings, as anxieties generated in patients tend to be different. Duration of outbreak can also become important and workarounds may be needed should there be prolonged cessation of services provided by external therapists, as this can interrupt patient recovery even for those who are not line listed.

The collaborative design of infection prevention and control strategies to manage outbreaks in a mental health setting cannot be overemphasized. IPAC must work closely with the unit to understand practices which may be contributing to transmission. Staff unfamiliar with outbreak management will require extra support to implement control measures and collect specimens. Patients also play a key role in understanding the outbreak and preventing further spread. Open and transparent communication in these outbreaks contributed to successful management.

\section{REFERENCES}

1. Ministry of Health and Long-Term Care. (2019) Infectious Diseases Protocol Appendix B: Provincial Case Definitions for Diseases of Public Health Significance: Disease: Respiratory Infection Outbreaks in Institutions and Public Hospitals. Retrieved from: http://www.health.gov.on.ca/en/pro/ programs/publichealth/oph_standards/ docs/respiratory_outbreaks_cd.pdf

2. Ministry of Health and Long-Term Care. (2019) Infectious Diseases Protocol Appendix B: Provincial Case Definitions for Diseases of Public Health Significance:

Disease: Gastroenteritis Outbreaks in Institutions and Public Hospitals. Retrieved from: http://www.health.gov. on.ca/en/pro/programs/publichealth/oph standards/docs/gastro_outbreaks_cd.pdf

3. Public Health Ontario. (2019) Labstract: Gastrointestinal Virus Testing Update. Retrieved from: https://www.publichealthontario.ca/-/ media/documents/lab/lab-sd-135gastrointestinal-virus-testing.pdf?la $=\mathrm{en}$

4. Gillbride, S. J., Lee B. E., Taylor, G. D., Forgie, S. E. (2009) Successful Containment of a Norovirus Outbreak in an Acute Adult Psychiatric Area. Infection Control and Hospital Epidemiology, 30(3), 289-291. DOI: 10.1086/595733

5. Government of Canada. (2019) Vaccination Coverage Goals and Vaccine Preventable Disease Reduction Targets by 2025 . Retrieved from: https://www. canada.ca/en/public-health/services/ immunization-vaccine-priorities/nationalimmunization-strategy/vaccinationcoverage-goals-vaccine-preventablediseases-reduction-targets-2025. html\#det22

6. Weber, D., Sickbert-Bennett, E. E., Vinjé, J., Brown, V. M., MacFarquhar, J. K., Engel, J.P., Rutala, W. A. (2003) Lessons Learned from a Norovirus Outbreak in a Locked Pediatric Inpatient Psychiatric Unit. Infection Control and Hospital Epidemiology, 26(10), 841-843 DOI: 10.1086/502504 